\title{
On the Spinor Representation of Surfaces in Euclidean 3-Space. *
}

\author{
Thomas Friedrich, Berlin
}

October 30, 2018

\section{Introduction}

The Weierstraß formula describes a conformal minimal immersion of a Riemann surface $M^{2}$ into the 3-dimensional Euclidean space $\mathbb{R}^{3}$. It expresses the immersion in terms of a holomorphic function $g$ and a holomorphic 1-form $\mu$ as the integral

$$
f=\operatorname{Re}\left(\int\left(1-g^{2}, i\left(1+g^{2}\right), 2 g\right) \mu\right): M^{2} \rightarrow \mathbb{R}^{3} .
$$

On the other hand let us consider the spinor bundle $S$ over $M^{2}$. This 2-dimensional vector bundle splits into

$$
S=S^{+} \oplus S^{-}=\Lambda^{0} \oplus \Lambda^{1,0}
$$

Therefore the pair $(g, \mu)$ can be considered as a spinor field $\varphi$ on the Riemann surface. The Cauchy-Riemann equation for $g$ and $\mu$ is equivalent to the homogeneous Dirac equation

$$
D(\varphi)=0
$$

The choice of the Riemannian metric in the fixed conformal class of $M^{2}$ is not essential since the kernel of the Dirac operator is a conformal invariant.

A similar description for an arbitrary surface $M^{2} \hookrightarrow \mathbb{R}^{3}$ is possible and has been pointed out probably for the first time by Eisenhardt (1909). This representation of any surface in $\mathbb{R}^{3}$ by a spinor field $\varphi$ on $M^{2}$ satisfying the inhomogeneous Dirac equation

$$
D(\varphi)=H \varphi
$$

involving the mean curvature $H$ of the surface has been used again in some recent papers (see $[\mathrm{KS}],[\mathrm{R}]$, [Tai1], [Tai2], [Tai3]). However, the mentioned authors describe the relationship between surfaces in $\mathbb{R}^{3}$ and solutions of the equation $(*)$ in local terms in order to get explicit formulas. The aim of the present paper is to clarify the mentioned representation of surfaces in $\mathbb{R}^{3}$ by solutions of the equation $D(\varphi)=$

\footnotetext{
${ }^{*}$ Supported by the SFB 288 of the DFG.
} 
$H \varphi$ in a geometrically invariant way. It turns out that the main idea leading to the description of a surface by a spinor field $\varphi$ is simple: Consider an immersion $M^{2} \hookrightarrow \mathbb{R}^{3}$ and fix a parallel spinor $\Phi$ on $\mathbb{R}^{3}$. Then the restriction $\varphi=\Phi_{\mid M^{2}}$ of $\Phi$ to the surface is (with respect to the inner geometry of $M^{2}$ ) a non-trivial spinor field on $M^{2}$ and defines a spinor $\varphi^{*}$ of constant length which is a solution of the inhomogeneous Dirac equation

$$
D\left(\varphi^{*}\right)=H \varphi^{*}
$$

Conversely, given a solution $\varphi$ of the equation $(*)$ with constant length there exists a symmetric endomorphism $E: T\left(M^{2}\right) \rightarrow T\left(M^{2}\right)$ such that the spinor field satisfies a "twistor type equation"

$$
\nabla_{X} \varphi=E(X) \cdot \varphi
$$

The resulting integrability conditions for the endomorphism $E$ are exactly the Gauß and Codazzi equations. As a consequence, the solution $\varphi$ of the Dirac equation

$$
D(\varphi)=H \varphi \quad, \quad|\varphi| \equiv \text { const }>0
$$

yields an isometric immersion of $M^{2}$ into $\mathbb{R}^{3}$. In a similar way one obtains the description of conformal immersions using the well-known formula for the transformation of the Dirac operator under a conformal change of the metric (see [BFGK]).

\section{The Dirac Operator of a Surface immersed into a Rie- mannian 3-Manifold.}

Let $Y^{3}$ be a 3-dimensional oriented Riemannian manifold with a fixed spin structure and denote by $M^{2}$ an oriented surface isometrically immersed into $Y^{3}$. Because the normal bundle of $M^{2}$ is trivial, the spin structure of $Y^{3}$ induces a spin structure on the Riemannian surface $M^{2}$. The spinor bundle $S$ of the 3 -manifold $Y^{3}$ yields by restriction the spinor bundle of the surface $M^{2}$. Over $M^{2}$ this bundle decomposes into

$$
S=S^{+} \oplus S^{-}
$$

where the subbundles $S^{ \pm}$are defined by (see $[\mathrm{F}]$ )

$$
S^{ \pm}=\left\{\varphi \in S: i \cdot e_{1} \cdot e_{2} \cdot \varphi= \pm \varphi\right\}
$$

Here $\left\{e_{1}, e_{2}\right\}$ denotes an oriented orthonormal frame in $T\left(M^{2}\right)$ and $X \cdot \varphi$ means the Clifford multiplication of a spinor $\varphi \in S$ by a vector $X \in T\left(M^{2}\right)$. Since in the 3-dimensional Clifford algebra the relation

$$
e_{1} \cdot e_{2}=e_{3}
$$

holds, we can replace the Clifford product $e_{1} \cdot e_{2}$ by the normal vector $\vec{N}$ of $M^{2} \hookrightarrow Y^{3}$ :

$$
S^{ \pm}=\{\varphi \in S: i \cdot \vec{N} \cdot \varphi= \pm \varphi\}
$$


Consider a spinor field $\Phi$ defined on the 3-manifold $Y^{3}$. Its restriction $\varphi=\Phi_{\mid M^{2}}$ is a spinor field defined on $M^{2}$ and decomposes therefore into $\varphi=\varphi^{+}+\varphi^{-}$with

$$
\varphi^{+}=\frac{1}{2}(\varphi+i \vec{N} \cdot \varphi) \quad, \quad \varphi^{-}=\frac{1}{2}(\varphi-i \vec{N} \cdot \varphi) .
$$

We denote by $\nabla^{Y^{3}}$ and $\nabla^{M^{2}}$ the covariant derivatives in the spinor bundles on $Y^{3}$ and $M^{2}$ respectively. For any vector $X \in T\left(M^{2}\right)$ we have the well-known formula (see $[\mathrm{BFGK}]$ )

$$
\nabla_{X}^{Y^{3}}(\Phi)=\nabla_{X}^{M^{2}}(\varphi)-\frac{1}{2}\left(\nabla_{X} \vec{N}\right) \cdot \vec{N} \cdot \varphi
$$

The vector $\left(\nabla_{X} \vec{N}\right)$ coincides with the second fundamental form II: $T\left(M^{2}\right) \rightarrow T\left(M^{2}\right)$ of the submanifold $M^{2} \hookrightarrow Y^{3}$. Since II is symmetric the Clifford product $e_{1} \cdot \operatorname{II}\left(e_{1}\right)+$ $e_{2} \cdot \operatorname{II}\left(e_{2}\right)$ is a scalar and equals $(-2 H)$ where $H$ denotes the mean curvature of the surface $M^{2}$. The Dirac operator $D$ of $M^{2}$ defined by the formula

$$
D(\varphi)=e_{1} \cdot \nabla_{e_{1}}^{M^{2}} \varphi+e_{2} \cdot \nabla_{e_{2}}^{M^{2}} \varphi
$$

can now be expressed by the covariant derivative $\nabla^{Y^{3}}$ and the mean curvature vector:

$$
e_{1} \cdot \nabla_{e_{1}}^{Y^{3}}(\Phi)+e_{2} \cdot \nabla_{e_{2}}^{Y^{3}}(\Phi)=D(\varphi)+H \cdot \vec{N} \cdot \varphi
$$

Suppose that the spinor field $\Phi$ on $Y^{3}$ is a real Killing spinor, i.e. there exists a number $\lambda \in \mathbb{R}^{1}$ such that for any tangent vector $\vec{T} \in T\left(Y^{3}\right)$ the derivative of $\Phi$ in the direction of $\vec{T}$ is given by the Clifford multiplication:

$$
\nabla_{\vec{T}}^{Y^{3}}(\Phi)=\lambda \cdot \vec{T} \cdot \Phi
$$

For the restriction $\varphi=\Phi_{\mid M^{2}}$ we obtain immediately the equation

$$
D(\varphi)=-2 \lambda \varphi-H \cdot \vec{N} \cdot \varphi .
$$

Using the decomposition $\varphi=\varphi^{+}+\varphi^{-}$the last equation is equivalent to the pair of equations

$$
D\left(\varphi^{+}\right)=(-2 \lambda-i H) \varphi^{-} \quad, \quad D\left(\varphi^{-}\right)=(-2 \lambda+i H) \varphi^{+} .
$$

We discuss two special cases.

Proposition 1: Let $M^{2}$ be a minimal surface in $Y^{3}$. Then the restriction $\varphi=\Phi_{\mid M^{2}}$ of any real Killing spinor $\Phi$ on $Y^{3}$ is an eigenspinor of constant length on the surface $M^{2}$ :

$$
D(\varphi)=-2 \lambda \varphi
$$

On the other hand, suppose that $\Phi$ is a parallel spinor $(\lambda=0)$ on $Y^{3}$. Then we obtain

$$
D\left(\varphi^{+}\right)=-i H \varphi^{-} \quad, \quad D\left(\varphi^{-}\right)=i H \varphi^{+} .
$$


If we introduce the spinor field $\varphi^{*}=\varphi^{+}-i \varphi^{-}$, a simple calculation shows

$$
D\left(\varphi^{*}\right)=H \varphi^{*}
$$

The spinor field $\varphi^{*}$ is given by

$$
\varphi^{*}=\varphi^{+}-i \varphi^{-}=\frac{1}{2}(\varphi+i \cdot \vec{N} \cdot \varphi)-\frac{i}{2}(\varphi-i \cdot \vec{N} \cdot \varphi)=\frac{1}{2}(1-i) \varphi+\frac{1}{2}(-1+i) \cdot \vec{N} \cdot \varphi .
$$

Moreover, the length of $\varphi^{*}$ is constant. This construction yields the

Proposition 2: $\quad$ Let $\Phi$ be a parallel spinor field defined on the 3-manifold $Y^{3}$ and denote by $\varphi=\Phi_{\mid M^{2}}$ its restriction to $M^{2}$. Define the spinor field $\varphi^{*}$ on $M^{2}$ by the formula

$$
\varphi^{*}=\frac{1}{2}(1-i) \varphi+\frac{1}{2}(-1+i) \cdot \vec{N} \cdot \varphi
$$

Then $\varphi^{*}$ is a spinor field of constant length on $M^{2}$ satisfying the Dirac equation

$$
D\left(\varphi^{*}\right)=H \varphi^{*}
$$

where $H$ denotes the mean curvature.

Remark 1: $\quad$ The map $\Phi \longmapsto \varphi^{*}$ associating to any parallel spinor $\Phi$ on $Y^{3}$ a solution of the equation $D\left(\varphi^{*}\right)=H \varphi^{*}$ is injective.

Remark 2: $\quad$ We can apply the above mentioned formulas not only for Killing spinors. Indeed, for any spinor field $\Phi$ we have

$$
D_{Y^{3}}(\Phi)=D(\varphi)+H \cdot \vec{N} \cdot \varphi+\vec{N} \cdot\left(\nabla_{\vec{N}}^{Y^{3}} \Phi\right)
$$

where $D_{Y^{3}}$ is the Dirac operator of the 3-manifold $Y^{3}$. Suppose there exists a function $\kappa: M^{2} \rightarrow \mathbb{C}$ such that the normal derivative $\left(\nabla_{\vec{N}}^{Y^{3}} \Phi\right)$ of the spinor field $\Phi$ is described by $\kappa$ :

$$
\left(\nabla_{\vec{N}}^{Y^{3}} \Phi\right)=\kappa \Phi
$$

Then we obtain

$$
D_{Y^{3}}(\Phi)=D(\varphi)+(H+\kappa) \vec{N} \cdot \varphi
$$

This formula (in arbitrary dimension) has been used for the calculation of the spectrum of the Dirac operator on hypersurfaces of the Euclidean space (see [Bä], [Tr1], $[\operatorname{Tr} 2])$. 


\section{Solutions of the Dirac Equation with Potential on Riemannian Surfaces.}

Let $\left(M^{2}, g\right)$ be an oriented, 2-dimensional Riemannian manifold with spin structure. $H: M^{2} \rightarrow \mathbb{R}^{1}$ denotes a given smooth, real-valued function defined on the surface. In this part we study spinor fields $\varphi$ on $M^{2}$ that are solutions of the differential equation

$$
D(\varphi)=H \varphi .
$$

If we decompose the spinor field into $\varphi=\varphi^{+}+\varphi^{-}$according to the splitting $S=$ $S^{+} \oplus S^{-}$of the spinor bundle the equation we want to study is equivalent to the system

$$
D\left(\varphi^{+}\right)=H \varphi^{-} \quad, \quad D\left(\varphi^{-}\right)=H \varphi^{+} .
$$

To any solution $\varphi$ of this equation we associate two forms $F_{ \pm}$defined for pairs $X, Y \in T\left(M^{2}\right)$ of tangent vectors:

$$
F_{+}(X, Y)=\operatorname{Re}\left(\nabla_{X} \varphi^{+}, Y \cdot \varphi^{-}\right) \quad, \quad F_{-}(X, Y)=\operatorname{Re}\left(\nabla_{X} \varphi^{-}, Y \cdot \varphi^{+}\right) .
$$

\section{Proposition 3:}

a.) $F_{ \pm}$are symmetric bilinear forms on $T\left(M^{2}\right)$.

b.) The trace of $F_{ \pm}$is given by $\operatorname{Tr}\left(F_{ \pm}\right)=-H\left|\varphi^{\mp}\right|^{2}$.

Proof: The symmetry of $F_{ \pm}$is a consequence of the Dirac equation as well as the assumption that $H$ is a real-valued function. Indeed, we have

$$
\begin{aligned}
\operatorname{Re}\left(\nabla_{e_{1}} \varphi^{+}, e_{2} \varphi^{-}\right) & =\operatorname{Re}\left(e_{1} \cdot \nabla_{e_{1}} \varphi^{+}, e_{1} \cdot e_{2} \cdot \varphi^{-}\right)=\operatorname{Re}\left(H \varphi^{-}-e_{2} \cdot \nabla_{e_{2}} \varphi^{+}, e_{1} \cdot e_{2} \cdot \varphi^{-}\right)= \\
& =H \cdot \operatorname{Re}\left(\varphi^{-}, e_{1} \cdot e_{2} \cdot \varphi^{-}\right)+\operatorname{Re}\left(\nabla_{e_{2}} \varphi^{+}, e_{2} \cdot e_{1} \cdot e_{2} \cdot \varphi^{-}\right)= \\
& =0+\operatorname{Re}\left(\nabla_{e_{2}} \varphi^{+}, e_{1} \cdot \varphi^{-}\right) .
\end{aligned}
$$

Moreover, we calculate the trace of $F_{ \pm}$:

$\operatorname{Tr}\left(F_{ \pm}\right)=\operatorname{Re}\left(\nabla_{e_{1}} \varphi^{ \pm}, e_{1} \cdot \varphi^{\mp}\right)+\operatorname{Re}\left(\nabla_{e_{2}} \varphi^{ \pm}, e_{2} \cdot \varphi^{\mp}\right)=-\operatorname{Re}\left(D\left(\varphi^{ \pm}\right), \varphi^{\mp}\right)=-H\left|\varphi^{\mp}\right|^{2}$.

We study now special solutions of the equation $D(\varphi)=H \varphi$, i.e. solutions with constant length $|\varphi| \equiv$ const $\neq 0$. It may happen that the components $\varphi^{ \pm}$have a non-empty zero set.

Proposition 4: Suppose that the spinor field $\varphi$ defined on the Riemannian surface $M^{2}$ is a solution of the equation

$$
D(\varphi)=H \varphi \quad \text { with }|\varphi| \equiv \text { const } \neq 0 .
$$

Then the forms $F_{ \pm}$are related by the equation 


$$
\left|\varphi^{+}\right|^{2} F_{+}=\left|\varphi^{-}\right|^{2} F_{-} .
$$

Proof: In case one of the spinors $\varphi^{+}$or $\varphi^{-}$vanishes at a fixed point $m_{0} \in M^{2}$ the relation between $F_{+}$and $F_{-}$is trivial. Otherwise there exists a neighbourhood $V$ of the point $m_{0} \in M^{2}$ such that both spinors $\varphi^{+}$and $\varphi^{-}$are not zero at any point $m \in V$. The spinors

$$
\frac{e_{1} \cdot \varphi^{-}}{\left|\varphi^{-}\right|} \quad, \quad \frac{e_{2} \cdot \varphi^{-}}{\left|\varphi^{-}\right|}
$$

are an orthonormal base in $S^{+}$with respect to the Euclidean scalar product $\operatorname{Re}(\cdot, \cdot)$. Therefore we obtain (on $V$ )

$$
\begin{aligned}
\nabla_{X} \varphi^{+} & =\operatorname{Re}\left(\nabla_{X} \varphi^{+}, \frac{e_{1} \cdot \varphi^{-}}{\left|\varphi^{-}\right|}\right) \frac{e_{1} \cdot \varphi^{-}}{\left|\varphi^{-}\right|}+\operatorname{Re}\left(\nabla_{X} \varphi^{+}, \frac{e_{2} \cdot \varphi^{-}}{\left|\varphi^{-}\right|}\right) \frac{e_{2} \cdot \varphi^{-}}{\left|\varphi^{-}\right|}= \\
& =\frac{1}{\left|\varphi^{-}\right|^{2}}\left\{F_{+}\left(X, e_{1}\right) e_{1}+F_{+}\left(X, e_{2}\right) e_{2}\right\} \cdot \varphi^{-} .
\end{aligned}
$$

A similar calculation yields the formula

$$
\nabla_{X} \varphi^{-}=\frac{1}{\left|\varphi^{+}\right|^{2}}\left\{F_{-}\left(X, e_{1}\right) e_{1}+F_{-}\left(X, e_{2}\right) e_{2}\right\} \cdot \varphi^{+}
$$

We multiply the equations by $\varphi^{+}$and $\varphi^{-}$respectively and sum up. Then we obtain

$$
\frac{1}{2} \nabla_{X}\left(\left|\varphi^{+}\right|^{2}+\left|\varphi^{-}\right|^{2}\right)=\operatorname{Re}\left(A(X) \varphi^{-}, \varphi^{+}\right)
$$

where the endomorphism $A: T\left(M^{2}\right) \rightarrow T\left(M^{2}\right)$ is defined by

$$
A(X)=\left\{\frac{F_{+}\left(X, e_{1}\right)}{\left|\varphi^{-}\right|^{2}}-\frac{F_{-}\left(X, e_{1}\right)}{\left|\varphi^{+}\right|^{2}}\right\} e_{1}+\left\{\frac{F_{+}\left(X, e_{2}\right)}{\left|\varphi^{-}\right|^{2}}-\frac{F_{-}\left(X, e_{2}\right)}{\left|\varphi^{+}\right|^{2}}\right\} e_{2} .
$$

Since $F_{ \pm}$are symmetric tensors, the endomorphism $A$ is symmetric too. Moreover, the trace of $A$ vanishes:

$$
\operatorname{Tr} A=\frac{1}{\left|\varphi^{-}\right|^{2}} \operatorname{Tr}\left(F_{+}\right)-\frac{1}{\left|\varphi^{+}\right|^{2}} \operatorname{Tr}\left(F_{-}\right)=-H+H=0 .
$$

The length of the spinor field $\varphi$ is constant. This implies

$$
\operatorname{Re}\left(A(X) \cdot \varphi^{-}, \varphi^{+}\right)=0 .
$$

At any point $m \in V$ of the set $V$ the spinors $\varphi^{+}, \varphi^{-}$are non-trivial. Then the rank of the endomorphisms $A: T\left(M^{2}\right) \rightarrow T\left(M^{2}\right)$ is not greater than one. All in all, $A$ is symmetric, $\operatorname{Tr}(A)=0$ and $\operatorname{rg}(A) \leq 1$, i.e. $A \equiv 0$.

We now consider the sum

$$
F=F_{+}+F_{-}
$$


At points with $\varphi^{+} \neq 0$ (or $\varphi^{-} \neq 0$ ) we have

$$
\frac{F}{|\varphi|^{2}}=\frac{F_{+}+F_{-}}{\left|\varphi^{+}\right|^{2}+\left|\varphi^{-}\right|^{2}}=\frac{\left(\frac{\left|\varphi^{-}\right|^{2}}{\left|\varphi^{+}\right|^{2}}+1\right) F_{-}}{\left|\varphi^{+}\right|^{2}+\left|\varphi^{-}\right|^{2}}=\frac{F_{-}}{\left|\varphi^{+}\right|^{2}}
$$

as well as

$$
\frac{F}{|\varphi|^{2}}=\frac{F_{+}+F_{-}}{\left|\varphi^{+}\right|^{2}+\left|\varphi^{-}\right|^{2}}=\frac{\left(1+\frac{\left|\varphi^{+}\right|^{2}}{\left|\varphi^{-}\right|^{2}}\right) F_{+}}{\left|\varphi^{+}\right|^{2}+\left|\varphi^{-}\right|^{2}}=\frac{F_{+}}{\left|\varphi^{-}\right|^{2}} .
$$

The endomorphism $E: T\left(M^{2}\right) \rightarrow T\left(M^{2}\right), E=\frac{F}{|\varphi|^{2}}$, is defined at all points of $M^{2}$ and the formulas derived in the proof of proposition 4 in fact prove the following

Proposition 5: Let $\varphi$ be a solution of the differential equation $D(\varphi)=H \varphi$ on a Riemannian surface $\left(M^{2}, g\right)$ with a real-valued function $H: M^{2} \rightarrow \mathbb{R}^{1}$. Suppose that the length $|\varphi| \equiv$ const $\neq 0$ of the spinor field $\varphi$ is constant. Then

$$
E(X)=\frac{1}{|\varphi|^{2}} \operatorname{Re}\left(\nabla_{X} \varphi, Y \cdot \varphi\right)
$$

is a symmetric endomorphism $E: T\left(M^{2}\right) \rightarrow T\left(M^{2}\right)$ such that

a.) $\nabla_{X} \varphi^{+}=E(X) \cdot \varphi^{-}, \quad \nabla_{X} \varphi^{-}=E(X) \cdot \varphi^{+}$

b.) $\operatorname{Tr}(E)=-H$.

For a given triple $\left(M^{2}, g, E\right)$ of a Riemannian surface and symmetric endomorphism the existence of a non-trivial solution $\varphi$ of the equation

$$
\nabla_{X} \varphi=E(X) \cdot \varphi
$$

implies certain integrability conditions. It turns out that in this way we obtain precisely the well-known Gauß and Codazzi equations of the classical theory of surfaces in Euclidean 3-space.

Proposition 6: Let $\left(M^{2}, g\right)$ be a 2-dimensional Riemannian surface with a fixed spin structure and suppose that $E: T\left(M^{2}\right) \rightarrow T\left(M^{2}\right)$ is a symmetric endomorphism. If there exists a non-trivial solution of the equation

$$
\nabla_{X} \varphi=E(X) \cdot \varphi \quad, \quad X \in T\left(M^{2}\right)
$$

then

a.) (Codazzi equation): $\quad \nabla_{X}(E(Y))-\nabla_{Y}(E(X))-E([X, Y])=0$.

b.) (Gauß equation): $\operatorname{det}(E)=\frac{1}{4} G, \quad$ where $G$ is the Gaussian curvature of $\left(M^{2}, g\right)$. 
Proof: We prove the two equations in a way similar to the derivation of the integrability conditions for the Riemannian metric in case the space admits a Killing spinor (see $[\mathrm{BFGK}])$. We differentiate the equation

$$
\nabla_{X} \varphi=E(X) \cdot \varphi
$$

and then we calculate the curvature tensor $R^{S}$ of the spinor bundle $S$ :

$$
\begin{aligned}
R^{S}(X, Y) \varphi & =\nabla_{X} \nabla_{Y} \varphi-\nabla_{Y} \nabla_{X} \varphi-\nabla_{[X, Y]} \varphi= \\
& =\left\{\nabla_{X}(E(Y))-\nabla_{Y}(E(X))-E([X, Y])+E(Y) E(X)-E(X) E(Y)\right\} \cdot \varphi .
\end{aligned}
$$

On the other side, the curvature tensor $R^{S}: S \rightarrow S$ is given by the formula

$$
R^{S}\left(e_{1}, e_{2}\right)=\frac{1}{2} R_{1212} e_{1} \cdot e_{2} .
$$

Denote by $A(X, Y)$ the differential of $E$ :

$$
A(X, Y)=\nabla_{X}(E(Y))-\nabla_{Y}(E(X))-E([X, Y]) .
$$

A simple algebraic calculation in the spin representation then leads to the equations

$$
\begin{gathered}
-A(X, Y) \varphi^{-}=\left(2 \operatorname{det}(E)+\frac{R_{1212}}{2}\right) i \varphi^{+} \\
A(X, Y) \varphi^{+}=\left(2 \operatorname{det}(E)+\frac{R_{1212}}{2}\right) i \varphi^{-} .
\end{gathered}
$$

We multiply the first equation once by the vector $A(X ; Y)$ :

$$
\|A(X, Y)\|^{2} \varphi^{-}=-\left(2 \operatorname{det}(E)+\frac{R_{1212}}{2}\right)^{2} \varphi^{-}
$$

and then we conclude $A(X, Y) \equiv 0$ (Codazzi equation) as well as $\operatorname{det}(E)=$ $-\frac{1}{4} R_{1212}=\frac{1}{4} G$ (Gauß equation).

For a given triple $\left(M^{2}, g, E\right)$ consisting of a Riemannian spin surface $\left(M^{2}, g\right)$ and of a symmetric endomorphism $E$ we will denote by $\mathcal{K}\left(M^{2}, g, E\right)$ the space of all spinor fields $\varphi$ satisfying the equation $\nabla_{X} \varphi=E(X) \cdot \varphi$. It is invariant under the quaternionic structure $\alpha: S \rightarrow S$, i.e. $\mathcal{K}\left(M^{2}, g, E\right)$ is a quaternionic vector space (see $\S 4)$. Denote by $(-H)$ the trace of $E$,

$$
\operatorname{Tr}(E)=-H \text {. }
$$

Then we have

$$
\mathcal{K}\left(M^{2}, g, E\right) \subset \operatorname{ker}(D-H) .
$$

In this part of the paper we prove that any spinor field $\varphi \in \operatorname{ker}(D-H)$ of constant length belongs to one of the subspaces $\mathcal{K}\left(M^{2}, g, E\right)$ for a suitable symmetric endomorphism $E, \operatorname{Tr}(E)=-H$. 
Finally, we consider the lengths

$$
L_{+}=\left\|\varphi^{+}\right\|^{2} \quad, \quad L_{-}=\left\|\varphi^{-}\right\|^{2}
$$

of a non-trivial solution $\varphi \in \mathcal{K}\left(M^{2}, g, E\right)$. Using the integrability condition $\operatorname{det}(E)=$ $\frac{G}{4}$ (i.e. $\|E\|^{2}=H^{2}-\frac{G}{2}$ ) as well as the well-known formula $D^{2}=\Delta+\frac{G}{2}$ for the square $D^{2}$ of the Dirac operator we can derive formulas for $\Delta\left(L_{ \pm}\right)$:

$$
\begin{aligned}
\Delta\left(L_{ \pm}\right) & =2\left(\Delta\left(\varphi^{ \pm}\right), \varphi^{ \pm}\right)-2\left\langle\nabla\left(\varphi^{ \pm}\right), \nabla\left(\varphi^{ \pm}\right)\right\rangle \\
& =2\left(D^{2}\left(\varphi^{ \pm}\right), \varphi^{ \pm}\right)-2\left(\frac{G}{2}\right) \bullet\left\|\varphi^{ \pm}\right\|^{2}-2\|E\|^{2}\left\|\varphi^{\mp}\right\|^{2} \\
& =2\left(H^{2}-\frac{G}{2}\right)\left(L_{ \pm}-L_{\mp}\right)+2 \operatorname{Re}\left(\operatorname{grad}(H) \cdot \varphi^{\mp}, \varphi^{ \pm}\right)
\end{aligned}
$$

In particular, if $H \equiv$ const is constant, the difference $u=L_{+}-L_{-}$satisfies the differential equation

$$
\Delta(u)=4\left(H^{2}-\frac{G}{2}\right) u
$$

\section{The Period Form of a Spinor with $\nabla_{X} \varphi=E(X) \cdot \varphi$.}

We consider a spinor field $\varphi$ on a Riemannian surface $\left(M^{2}, g\right)$ such that

$$
\nabla_{X} \varphi=E(X) \cdot \varphi
$$

for a fixed symmetric endomorphism $E$. The spinor bundle $S$ carries a quaternionic structure $\alpha: S \rightarrow S$ commuting with Clifford multiplication and interchanging the decomposition $S=S^{+} \oplus S^{-}$(see [F]). For any spinor field $\varphi=\varphi^{+}+\varphi^{-}$we define three 1 -forms by

$$
\begin{gathered}
\xi^{\varphi}(X)=2\left(X \cdot \varphi^{+}, \varphi^{-}\right) \\
\xi_{+}^{\varphi}(X)=\left(X \cdot \varphi^{+}, \alpha\left(\varphi^{+}\right)\right) \quad, \quad \xi_{-}^{\varphi}(X)=\left(X \cdot \varphi^{-}, \alpha\left(\varphi^{-}\right)\right) .
\end{gathered}
$$

$\xi^{\varphi}$ and $\xi_{+}^{\varphi}$ are $\Lambda^{1,0}{ }_{-}$forms, $\xi_{-}^{\varphi}$ is a $\Lambda^{0,1}$-form. Indeed, $e_{1} \cdot e_{2}$ acts on $S^{+}$(on $S^{-}$) by multiplication by $(-i)$ (by $i$ ). Now we obtain

$$
\left(\star \xi^{\varphi}\right)\left(e_{1}\right)=-\xi^{\varphi}\left(e_{2}\right)=2\left(-e_{2} \cdot \varphi^{+}, \varphi^{-}\right)=\left(-i e_{2} \cdot e_{1} \cdot e_{2} \cdot \varphi^{+}, \varphi^{-}\right)=-i \xi^{\varphi}\left(e_{1}\right),
$$

i.e. $\star \xi^{\varphi}=-i \xi^{\varphi}$ holds. A similar calculation gives $\star \xi_{+}^{\varphi}=-i \xi_{+}^{\varphi}$ and $\star \xi_{-}^{\varphi}=i \xi_{-}^{\varphi}$. We split the 1-form $\xi^{\varphi}$ into its real and imaginary part:

$$
\xi^{\varphi}=w^{\varphi}+i \mu^{\varphi} .
$$

Moreover, we introduce the 1-form $\Omega^{\varphi}$

$$
\Omega^{\varphi}=\xi_{+}^{\varphi}-\xi_{-}^{\varphi} .
$$

Then we have

Proposition 7: $\quad$ Let $\left(M^{2}, g\right)$ be a Riemannian spin surface and $E: T\left(M^{2}\right) \rightarrow$ $T\left(M^{2}\right)$ a symmetric endomorphism of trace $-H$. Suppose the spinor field $\varphi$ is a solution of the equation $\nabla_{X} \varphi=E(X) \cdot \varphi$. Then 

a.) $d w^{\varphi}=0$.
b.) $d \mu^{\varphi}=2 H\left\{\left|\varphi^{-}\right|^{2}-\left|\varphi^{+}\right|^{2}\right\} d M^{2}$.
c.) $d \Omega^{\varphi}=0$.

Proof: We calculate $d w^{\varphi}$ :

$$
\begin{aligned}
\frac{1}{2} d w^{\varphi}(X, Y) & =X\left(\operatorname{Re}\left(Y \cdot \varphi^{+}, \varphi^{-}\right)\right)-Y\left(\operatorname{Re}\left(X \cdot \varphi^{+}, \varphi^{-}\right)\right)-\operatorname{Re}\left([X, Y] \cdot \varphi^{+}, \varphi^{-}\right)= \\
& =\{g(X, E(Y))-g(Y, E(X))\}\left|\varphi^{-}\right|^{2}+\{g(X, E(Y))-g(Y, E(X))\}\left|\varphi^{+}\right|^{2} .
\end{aligned}
$$

Since $E$ is symmetric, we obtain $d w^{\varphi}=0$. A similar calculation shows the formula for $d \mu^{\varphi}$. For the proof of $d \Omega^{\varphi}=0$ we first remark that the quaternionic structure $\alpha: S \rightarrow S$ and the hermitian product $(\cdot, \cdot)$ on $S$ are related by

$$
\left(\varphi_{1}, \alpha\left(\varphi_{2}\right)\right)=-\left(\overline{\alpha\left(\varphi_{1}\right), \varphi_{2}}\right)
$$

Using this formula we can transform $d \xi_{-}^{\varphi}$ in the following way:

$$
\begin{aligned}
d \xi_{-}^{\varphi}(X, Y)= & \left(Y \cdot E(X) \cdot \varphi^{+}, \alpha\left(\varphi^{-}\right)\right)+\left(Y \cdot \varphi^{-}, \alpha\left(E(X) \cdot \varphi^{+}\right)\right) \\
& -\left(X \cdot E(Y) \cdot \varphi^{+}, \alpha\left(\varphi^{-}\right)\right)-\left(X \cdot \varphi^{-}, \alpha\left(E(Y) \cdot \varphi^{+}\right)\right) \\
= & -\left(\overline{\alpha\left(Y \cdot E(X) \cdot \varphi^{+}\right), \varphi^{-}}\right)-\left(E(X) \cdot Y \cdot \varphi^{-}, \alpha\left(\varphi^{+}\right)\right) \\
& +\left(\overline{\alpha\left(X \cdot E(Y) \cdot \varphi^{+}\right), \varphi^{-}}\right)+\left(E(Y) \cdot X \cdot \varphi^{-}, \alpha\left(\varphi^{+}\right)\right) \\
= & -\left(E(X) \cdot Y \cdot \varphi^{-}, \alpha\left(\varphi^{+}\right)\right)-\left(E(X) \cdot Y \cdot \varphi^{-}, \alpha\left(\varphi^{+}\right)\right) \\
& +\left(E(Y) \cdot X \cdot \varphi^{-}, \alpha\left(\varphi^{+}\right)\right)+\left(E(Y) \cdot X \cdot \varphi^{-}, \alpha\left(\varphi^{+}\right)\right) .
\end{aligned}
$$

On the other hand we calculate $d \xi_{+}^{\varphi}$ :

$$
\begin{aligned}
d \xi_{+}^{\varphi}(X, Y)= & \left(Y \cdot E(X) \cdot \varphi^{-}, \alpha\left(\varphi^{+}\right)\right)+\left(Y \cdot \varphi^{+}, \alpha\left(E(X) \cdot \varphi^{-}\right)\right) \\
& -\left(X \cdot E(Y) \cdot \varphi^{-}, \alpha\left(\varphi^{+}\right)\right)-\left(X \cdot \varphi^{+}, \alpha\left(E(Y) \cdot \varphi^{-}\right)\right)= \\
= & \left(Y \cdot E(X) \cdot \varphi^{-}, \alpha\left(\varphi^{+}\right)\right)-\left(E(X) \cdot Y \cdot \varphi^{+}, \alpha\left(\varphi^{-}\right)\right) \\
& -\left(X \cdot E(Y) \cdot \varphi^{-}, \alpha\left(\varphi^{+}\right)\right)+\left(E(Y) \cdot X \cdot \varphi^{+}, \alpha\left(\varphi^{-}\right)\right) .
\end{aligned}
$$

Finally we obtain

$$
\begin{aligned}
d\left(\xi_{-}^{\varphi}-\xi_{+}^{\varphi}\right)(X, Y)= & -\left(\{E(X) \cdot Y+Y \cdot E(X)\} \varphi^{-}, \alpha\left(\varphi^{+}\right)\right) \\
& +\left(\{E(Y) \cdot X+X \cdot E(Y)\} \varphi^{-}, \alpha\left(\varphi^{+}\right)\right)- \\
= & 2\{g(E(X), Y)-g(E(Y), X)\}\left(\varphi^{-}, \alpha\left(\varphi^{+}\right)\right)
\end{aligned}
$$

and $d\left(\xi_{-}^{\varphi}-\xi_{+}^{\varphi}\right)=0$ follows again by the symmetry of $E$.

Let us consider the case that $\left(M^{2}, g\right)$ is isometrically immersed into the Euclidean space $\mathbb{R}^{3}, \Phi$ is a parallel spinor on $\mathbb{R}^{3}$ and the spinor field $\varphi^{*}$ on $M^{2}$ defined by the formula 


$$
\varphi^{*}=\frac{1}{2}\left(\Phi_{\mid M^{2}}+i \cdot \vec{N} \cdot \Phi_{\mid M^{2}}\right)+\frac{i}{2}\left(i \cdot \vec{N} \cdot \Phi_{\mid M^{2}}-\Phi_{\mid M^{2}}\right)
$$

(see $\S 2$ ). In this case the forms $w^{\varphi^{*}}$ and $\Omega^{\varphi^{*}}$ are given by the expressions

$$
w^{\varphi^{*}}(X)=-\operatorname{Im}(X \cdot \Phi, \Phi) \quad, \quad \Omega^{\varphi^{*}}(X)=(X \cdot \Phi, \alpha(\Phi)),
$$

and are exact 1 -forms. Indeed, we defined functions $f: \mathbb{R}^{3} \rightarrow \mathbb{R}^{1}$ and $g: \mathbb{R}^{3} \rightarrow \mathbb{C}$ by

$$
f(m)=-\operatorname{Im}\langle m \cdot \Phi, \Phi\rangle \quad, \quad g(m)=\langle m \cdot \Phi, \alpha(\Phi)\rangle
$$

and then we have $d f=w^{\varphi^{*}}, d g=\Omega^{\varphi^{*}}$. We remark that $f$ and $g$ describe in fact the isometric immersion $M^{2} \hookrightarrow \mathbb{R}^{3}$ we started with. The 3-dimensional spinor $\Phi \in \Delta_{3}$ defines a real 3-dimensional subspace $\Delta_{3}(\Phi)$ by

$$
\Delta_{3}(\Phi)=\left\{\Psi \in \Delta_{3}: \quad \operatorname{Re}(\Psi, \Phi)=0\right\} .
$$

The map $\Psi \rightarrow(-\operatorname{Im}(\Psi, \Phi),(\Psi, \alpha(\Phi)))$ is an isometry between $\Delta_{3}(\Phi)$ and $\mathbb{R}^{1} \oplus \mathbb{C}=$ $\mathbb{R}^{3}$. Clearly, the immersion $M^{2} \hookrightarrow \mathbb{R}^{3}$ is given by

$$
M^{2} \ni m \longmapsto m \cdot \Phi \in \Delta_{3}(\Phi)
$$

i.e. by the functions $f_{\mid M^{2}}$ and $g_{\mid M^{2}}$. With respect to $d\left(f_{\mid M^{2}}\right)=w^{\varphi^{*}}$ and $d\left(g_{\mid M^{2}}\right)=$ $\Omega^{\varphi^{*}}$ we obtain a formula for the isometric immersion $M^{2} \hookrightarrow \mathbb{R}^{3}$ :

$$
\oint\left(w^{\varphi^{*}}, \Omega^{\varphi^{*}}\right): M^{2} \rightarrow \mathbb{R}^{3} .
$$

(Weierstraß representation of the surface.)

In general, we call a solution $\varphi$ of the differential equation $\nabla_{X} \varphi=E(X) \cdot \varphi$ exact iff the corresponding forms $w^{\varphi}, \Omega^{\varphi}$ are exact 1-forms. Using the definition

$$
\operatorname{Hess}(h)(X, Y)=\frac{1}{2}\left\{g\left(\nabla_{X}(\operatorname{grad}(h)), Y\right)+g\left(X, \nabla_{Y}(\operatorname{grad}(h))\right)\right\}
$$

of the Hessian of a smooth function $h$ defined on a Riemannian manifold we obtain the

Proposition 8: Let $\varphi \in \mathcal{K}\left(M^{2}, g, E\right)$ be an exact solution of the differential equations $\nabla_{X} \varphi=E(X) \cdot \varphi$ with $d f=w^{\varphi}, d g=\Omega^{\varphi}$. Then

a.) $\operatorname{Hess}(f)=2\left(\left|\varphi^{+}\right|^{2}-\left|\varphi^{-}\right|^{2}\right) E$.

b.) $|\operatorname{grad} f|^{2}=4\left|\varphi^{+}\right|^{2}\left|\varphi^{-}\right|^{2}$.

c.) $\operatorname{Hess}(g)=-4\left(\varphi^{-}, \alpha\left(\varphi^{+}\right)\right) E$.

d.) $|\operatorname{grad}(g)|^{2}=\left(\left|\varphi^{+}\right|^{2}-\left|\varphi^{-}\right|^{2}\right)^{2}$. 
In particular, the determinant of the Hessian of the function $f$ is given by

$$
\operatorname{det}(H e s s(f))=4\left(\left|\varphi^{+}\right|^{2}-\left|\varphi^{-}\right|^{2}\right)^{2} \operatorname{det}(E)=\left(\left|\varphi^{+}\right|^{2}-\left|\varphi^{-}\right|^{2}\right)^{2} G .
$$

Here we used proposition 6 , i.e. $\operatorname{det}(E)=\frac{1}{4} G$.

Corollary: Let $M^{2}$ be a compact Riemannian spin-manifold and suppose that $\varphi \in \mathcal{K}\left(M^{2}, g, E\right)$ is an exact, non-trivial solution. Then the spinors $\varphi^{+}$or $\varphi^{-}$vanish at least at out one point. Moreover, there exists $m_{0} \in M^{2}$ such that $G\left(m_{0}\right) \geq 0$.

Proof: At a maximum point $m_{0} \in M^{2}$ of $f$ we have

$$
\operatorname{grad}(f)\left(m_{0}\right)=0 \quad, \quad \operatorname{det}\left(\operatorname{Hess}(f)\left(m_{0}\right)\right) \geq 0 \text {. }
$$

Recall that for any 2-dimensional Riemannian manifold $\left(M^{2}, g\right)$ and any function $h: M^{2} \rightarrow \mathbb{R}^{1}$ the 2 -form

$$
\left\{2 \operatorname{det}(\operatorname{Hess}(h))-|\operatorname{grad}(h)|^{2} G\right\} d M^{2}=d \mu^{1}
$$

is exact (see [S], page 47). Using this formula in case of an exact solution $\varphi \in$ $\mathcal{K}\left(M^{2}, g, E\right)$ we obtain

$$
\int_{M^{2}}\left(\left|\varphi^{+}\right|^{2}-\left|\varphi^{-}\right|^{2}\right)^{2} G=2 \int_{M^{2}}\left|\varphi^{+}\right|^{2}\left|\varphi^{-}\right|^{2} G .
$$

Corollary: Let $M^{2}$ be a compact Riemannian spin manifold and suppose that $\varphi \in \mathcal{K}\left(M^{2}, g, E\right)$ is an exact solution. Then

$$
\int_{M^{2}}\left(|\varphi|^{4}-6\left|\varphi^{+}\right|^{2}\left|\varphi^{-}\right|^{2}\right) G=0 .
$$

We again discuss the last formula in case of an isometrically immersed surface $M^{2} \hookrightarrow$ $\mathbb{R}^{3}$ and a given parallel spinor $\Phi$ on $\mathbb{R}^{3}$. We apply the integral formula to the spinor $\varphi^{*}=\varphi_{+}^{*}+\varphi_{-}^{*}$ where

$$
\varphi_{+}^{*}=\frac{1}{2}(\Phi+i \vec{N} \cdot \Phi) \quad, \quad \varphi_{-}^{*}=\frac{1}{2}(-\Phi+i \cdot \vec{N} \cdot \Phi) .
$$

In this case we have

$$
\left|\varphi_{+}^{*}\right|^{2}=\frac{1}{2}|\Phi|^{2}+\frac{1}{2}\langle i \vec{N} \cdot \Phi, \Phi\rangle \quad, \quad\left|\varphi_{-}^{*}\right|^{2}=\frac{1}{2}|\Phi|^{2}-\frac{1}{2}\langle i \vec{N} \cdot \Phi, \Phi\rangle
$$

and $(|\Phi| \equiv 1)$ therefore

$$
1-6\left|\varphi_{+}^{*}\right|^{2}\left|\varphi_{-}^{*}\right|^{2}=-\frac{1}{2}+\frac{3}{2}\langle i \cdot \vec{N} \cdot \Phi, \Phi\rangle^{2} .
$$


Consequently the integral formula yields

$$
\int_{M^{2}} G=3 \int_{M^{2}}\langle i \vec{N} \Phi, \Phi\rangle^{2} G .
$$

The spinors $i \Phi$ as well as $\vec{N} \cdot \Phi$ belong to $V(\Phi) \subset \Delta_{3}$, the space of the immersion $M^{2} \hookrightarrow \mathbb{R}^{3}=V(\Phi)$. The last formula means therefore

$$
\int_{M^{2}} G=3 \int_{M^{2}}\left\langle\vec{N}, \mathfrak{a}_{3}\right\rangle^{2} G
$$

for the unit vector $\mathfrak{a}_{3}=i \Phi \in V(\Phi)=\mathbb{R}^{3}$.

\section{The Spin Formulation of the Theory of Surfaces in $\mathbb{R}^{3}$.}

An oriented, immersed surface $M^{2} \hookrightarrow \mathbb{R}^{3}$ inherits from $\mathbb{R}^{3}$ an inner metric $g$, a spin structure and a solution $\varphi$ of the Dirac equation

$$
D(\varphi)=H \varphi
$$

of constant length $|\varphi| \equiv 1$ where $H$ denotes the mean curvature of the surface. The spinor field $\varphi$ on $M^{2}$ is the restriction of a parallel spinor field $\Phi$ of the Euclidean space $\mathbb{R}^{3}$. The period forms $w^{\varphi}$ and $\Omega^{\varphi}$ are exact and the immersion $M^{2} \hookrightarrow \mathbb{R}^{3}$ is given by integration of the $\mathbb{R}^{1} \oplus \mathbb{C}=\mathbb{R}^{3}$ valued form $\left(w^{\varphi}, \Omega^{\varphi}\right)$. At least locally the converse is true: Given an oriented, 2-dimensional Riemannian manifold $\left(M^{2}, g\right)$ with a fixed spin structure and a solution of constant length of the Dirac equation $D(\varphi)=H \varphi$ for some smooth function $H: M^{2} \rightarrow \mathbb{R}^{1}$, there exists a symmetric endomorphism $E: T\left(M^{2}\right) \rightarrow T\left(M^{2}\right)$ such that $\varphi \in \mathcal{K}\left(M^{2}, g, E\right)$. Moreover, $2 E$ is the second fundamental form of an isometric immersion $\left(M^{2}, g\right) \rightarrow \mathbb{R}^{3}$. We formulate this description of the theory of surfaces in $\mathbb{R}^{3}$ in the following

Theorem 1: Let $\left(M^{2}, g\right)$ be an oriented, 2-dimensional Riemannian manifold and $H: M^{2} \rightarrow \mathbb{R}^{1}$ a smooth function. Then there is a correspondence between the following datas:

1. an isometric immersion $\left(\tilde{M}^{2}, g\right) \rightarrow \mathbb{R}^{3}$ of the universal covering $\tilde{M}^{2}$ into the Euclidean space $\mathbb{R}^{3}$ with mean curvature $H$.

2. a solution $\varphi$ with constant length $|\varphi| \equiv 1$ of the Dirac equation $D(\varphi)=H \cdot \varphi$.

3. a pair $(\varphi, E)$ consisting of a symmetric endomorphism $E$ such that $\operatorname{Tr}(E)=-H$ and a spinor field $\varphi$ satisfying the equation $\nabla_{X} \varphi=E(X) \cdot \varphi$.

We apply now the well-known formulas for the change of the Dirac operator under a conformal change of the metric. Suppose that $\tilde{g}=\sigma g$ are two conformally equivalent metrics on $M^{2}$ where $\sigma: M^{2} \rightarrow(0, \infty)$ is a positive function. Denote by $D$ and $\tilde{D}$ the Dirac operator corresponding to the metric $g$ and $\tilde{g}$ respectively. Then 


$$
\tilde{D}(\tilde{\varphi})=\sigma^{-3 / 4} \widetilde{D\left(\sigma^{1 / 4} \varphi\right)}
$$

holds (see $[\mathrm{BFGK}]$ ). Let us consider a solution $\varphi$ of the Dirac equation

$$
D(\varphi)=\lambda \varphi
$$

on $\left(M^{2}, g\right)$ and suppose that $\varphi$ never vanishes. We introduce the Riemannian metric $\tilde{g}=|\varphi|^{4} g$ as well as the spinor field $\varphi^{*}=\frac{\varphi}{|\varphi|}$. Then we obtain

$$
\tilde{D}\left(\varphi^{*}\right)=\frac{\lambda}{|\varphi|^{2}} \varphi^{*} \quad, \quad\left|\varphi^{*}\right| \equiv 1,
$$

and thus an isometric immersion $\left(\tilde{M}^{2},|\varphi|^{4} g\right) \rightarrow \mathbb{R}^{3}$ with mean curvature $H=\frac{\lambda}{|\varphi|^{2}}$.

Theorem 2: $\quad$ Let $\left(M^{2}, g\right)$ be an oriented, 2-dimensional Riemannian manifold. Any spinor field $\varphi$ without zeros that is a solution of the equation

$$
D(\varphi)=\lambda \varphi
$$

defines an isometric immersion $\left(\tilde{M}^{2},|\varphi|^{4} g\right) \hookrightarrow \mathbb{R}^{3}$ with mean curvature $H=\frac{\lambda}{|\varphi|^{2}}$.

Remark 1: Consider the case that $M^{2} \hookrightarrow S^{3}$ is a minimal surface in $S^{3}$. Let $\Phi$ be a real Killing spinor on $S^{3}$, i.e.

$$
\nabla_{\vec{T}}(\Phi)=\frac{1}{2} \vec{T} \cdot \Phi
$$

The restriction $\varphi=\Phi_{\mid M^{2}}$ is an eigenspinor of the Dirac operator on $M^{2}$ with constant length (proposition 1). Therefore $\varphi$ defines an isometric immersion of $\left(\tilde{M}^{2}, g\right) \hookrightarrow \mathbb{R}^{3}$ with mean curvature $H \equiv-1$. This transformation associates to any minimal surface $M^{2} \hookrightarrow S^{3}$ a surface of constant mean curvature $H \equiv-1$ in $\mathbb{R}^{3}$, a well-known construction (see $[\mathrm{La}]$ ).

Remark 2: Using the described correspondence between isometric immersions of surfaces into $\mathbb{R}^{3}$ and solutions of the Dirac equation $D(\varphi)=H \cdot \varphi$ one can immediately remark that several statements of the elementary theory of surfaces are equivalent to several statements concerning solutions of the twistor equation (see [BFGK]). For example, in [Li] (see also proposition 6) one can find the following theorem: if $f: M^{2} \rightarrow \mathbb{R}^{1}$ is a real-valued function such that the equation

$$
\nabla_{\vec{T}}(\varphi)+\frac{1}{2} f \cdot \vec{T} \cdot \varphi=0
$$

admits a non-trivial solution then $f$ is constant and $f^{2}=G$. In the theory of surfaces this statement correspondends to the fact that an umbilic surface is a part of the sphere or the plane. Indeed, an umbilic surface $M^{2} \hookrightarrow \mathbb{R}^{3}$ admits a spinor field $\varphi$ such that 


$$
\nabla_{\vec{T}}(\varphi)+\frac{1}{2} H \vec{T} \cdot \varphi=0
$$

and therefore $H^{2}=G=$ const, i.e. the second fundamental form is proportional to the metric. In a similar way one can translate other facts of the theory of surfaces into properties of solutions of the equation $\nabla_{X} \varphi=E(X) \cdot \varphi$.

\section{References}

[Bä] C. Bär. Extrinsic bounds for eigenvalues of the Dirac operator, Preprint Universität Freiburg, Oktober 1997.

[BFGK] H. Baum, Th. Friedrich, R. Grunewald, I. Kath. Twistors and Killing spinors on Riemannian manifolds, Teubner-Verlag Leipzig / Stuttgart 1991.

[F] Th. Friedrich. Dirac-Operatoren in der Riemannschen Geometrie, ViewegVerlag Braunschweig/ Wiesbaden 1997.

[KPP] G. Kamberov, F. Pedit, U. Pinkall. Bonnet pairs and isothermic surfaces, Preprint dg-ga 9610006.

[KS] R. Kussner, N. Schmitt. Representation of surfaces in space, Preprint dgga 9610005 .

[La] H.B. Lawson. The global behaviour of minimal surfaces in $S^{n}$, Ann. Math. 1970, V. 92, No.2, 224-237.

[Li] A. Lichnerowicz. Spin manifolds, Killing spinors and universality of the Hijazi inequality, Lett. Math. Phys. 13 (1987), 331-344.

[R] J. Richter. Conformal maps of a Riemannian surface into the space of quaternions, Dissertation TU Berlin 1997.

[S] A. Svec. Global differential geometry of surfaces, Verlag der Wissenschaften, Berlin 1981.

[Tai1] I.A. Taimanov. Surfaces of revolution in terms of solutions, Ann. Glob. Anal. Geom. 15 (1997), 419-435.

[Tai2] I.A. Taimanov. Modified Novikov-Veselov equation and differential geometry of surfaces, Transl. Amer. Math. Soc., Ser. 2, V. 179 (1997), 133-151.

[Tai3] I.A. Taimanov. The Weierstraß representation of closed surfaces in $\mathbb{R}^{3}$, Preprint SFB 288, No. 291, TU Berlin (1997), dg-ga 9710029.

[Tr1] A. Trautman. Spinors and the Dirac operator on hypersurfaces I. General Theory, Journ. Math. Phys. 33 (1992), 4011-4019.

[Tr2] A. Trautman. The Dirac operator on hypersurfaces, Acta Phys. Polon. B 26 (1995), 1283-1310. 
Thomas Friedrich

Humboldt-Universität zu Berlin

Institut für Reine Mathematik

Sitz: Ziegelstraße 13a

D-10099 Berlin

e-mail: friedric@mathematik.hu-berlin.de 